\title{
The Effect of Human Recombinant Tumor Necrosis Factor Receptor-2 on Reducing Inflammatory of Collagen -Induced Arthritis in Balb/c Mice
}

\author{
Shahla Korani ${ }^{1}$, Bahram Kazemi ${ }^{2,3}$, Adel Haghighi ${ }^{4}$, Amin Reza Nikpoor ${ }^{5}$, Mojgan Bandehpour ${ }^{1,2,3, *}$ \\ ${ }^{1}$ Department of Biotechnology, School of Advanced Technologies in Medicine, Shahid Beheshti University of Medical \\ Sciences, Tehran, Iran \\ ${ }^{2}$ Cellular and Molecular Biology Research Center, Shahid Beheshti University of Medical Sciences, Tehran, Iran \\ ${ }^{3}$ Department of Biotechnology, School of Medicine, Shahid Beheshti University of Medical Sciences, Tehran, Iran \\ ${ }^{4}$ Department of Pathology, Faculty of Specialized Veterinary Sciences, Sciences \& Researches Branch Islamic Azad \\ University, Tehran, Iran \\ ${ }^{5}$ Department of Immunology, Faculty of Medicine, Hormozgan University of Medical Sciences, Bandar Abbas, Iran \\ * Corresponding author: Mojgan Bandehpour, Cellular and Molecular Biology Research Center, Shahid Beheshti University of Medical Sciences, Tehran, \\ Iran. Tel/Fax: +98-21-22439957; E-mail: m.bandehpour@sbmu.ac.ir
}

\begin{abstract}
Background: The tumor necrosis factor alpha $(\mathrm{TNF} \alpha)$ is a cytokine that produced principally by monocyte/macrophages and Tlymphocytes, respectively. TNF $\alpha$ is recognized as the primary mediator of immunity in inflammation reaction. One important application of Tumor Necrosis Factor Receptor 2 (TNFR2) is for the treatment of autoimmune diseases like rheumatoid arthritis (RA).

Objectives: The aim of this study is to examine the therapeutic trace of the recombinant humanTNFR2 on collageninduced arthritis (CIA) in mice.

Materials and Methods: CIA was created in 20 mice by immunization with bovine type II collagen (CII). After the mice were boosted on day 21 , they were injected with the recombinant protein in test group (1 mg.kg-1) and assessed edema in paws and knee joints after two weeks. The quantities of inflammatory cytokines such as TNF- $\alpha$, interleukin-1 beta (IL- $\beta 1$ ), interleukin-6 (IL-6), and interleukin-10(IL-10) in serum were evaluated through enzyme-linked immunosorbent assay (ELISA) kit. In addition, the histopathology of joints sections was analyzed.

Results: The cytokines TNF- $\alpha$, IL- $1 \beta$, and IL- 6 values in serum markedly decreased in groups treated with TNFR2 (P $<0.01-0.001)$. The results showed that treatment with TNFR2 significantly reduced edema in paws and joints $(\mathrm{P}<$ 0.01-0.001).

Conclusions: Pathological investigations proved that administration of recombinant TNF receptor has blocked or protected joints from progressive damage. This study suggests that the anti-arthritic effectiveness of TNFR2 will repress the symptoms of rheumatoid arthritis. Moreover, it seems that TNFR2 is a strong candidate for the treatment of the RA disease.
\end{abstract}

Keywords: ADAM17 Protein; Cytokines; Serpinh1 protein, mouse, Humans; Tumor Necrosis Factor-alpha

\section{Background}

Rheumatoid arthritis is a common autoimmune disease that is related to advanced disability, systemic inflammation, and early death (1). Although the cause of rheumatoid arthritis is poorly known, proinflammatory cytokines such as tumor necrosis factor $\alpha$ $(\mathrm{TNF} \alpha)$, as a mediator protein in the immune system, play an important role in the pathogenesis of rheumatoid arthritis $(2,3)$.TNF as a signaling molecule controls intercellular communication that mediates inflammation, apoptosis, immunoregulation, and cellular proliferation/differentiation (4). The main role of TNF- $\alpha$ is in the induction of inflammation and immunity through activating immune cells in reaction to infections and tissue injury. The moderated expression of TNF is therefore fundamental to attack infections and raise tissue repair. However, the dysregulation of TNF production and signaling can lead to chronic inflammation, which may uncontrolled inflammation and autoimmune disease (5). Signal transduction is mediated- through two distinct receptors TNFR1 
(TNFRSF1, CD120a) and TNFR2 (TNFRSF2, CD120b). TNFR1 is ubiquitously expressed in nearly all tissue cells, probably due to the extensive activity range of TNF's. TNFR2 is expressed in a limited manner on certain cells of the immune system, including $\mathrm{T}$ regulatory cells (Tregs), endothelial cells, and neuronal tissues and its expression can be greatly controlled by the cellular function and cell type. Both receptors are type I transmembrane glycoproteins with extracellular and intracellular components. Binding of TNF to TNFRs leads to signals transducion through both receptors. The current progress in the understanding of the pathogenetic mechanisms of rheumatoid arthritis and use of genetic engineering techniques have resulted in designing new-biologic drugs such as TNFRs that are able to inhibit the function of several cytokines $(6,7)$ In this study, we survey the cloning and the expression of a TNF receptor2 in Chinese hamster ovary $(\mathrm{CHO})$ cells, as well as purifying recombinant protein used in the treatment of collagen-induced arthritis (CIA) in Balb/c mice.

\section{Objectives}

The aim of this study was to evaluate the therapeutic effectuality of the recombinant human TNFR2 on collagen-induced arthritis (CIA) in mice. We have proposed that arthritis symptoms would be decreased with the receptor-ligand complex constitution between rTNFR 2 and TNF- $\alpha$, consequently the TNF- $\alpha$ cytokine will be reduced in synovial fluid.

\section{Materials and Methods}

\subsection{Animals}

Female Balb/c mice (Inbred strain of mouse) of 16-20 week age were obtained from Razi Institute (Karaj, Iran). Mice were keeped under standard conditions. All experiments were performed according to the Animal Care and Use Protocol of Shahid Beheshti University of Medical Sciences (Tehran, Iran) and approved by the Ethical Committee of the University.

\subsection{Expression, and Purification of a TNF Receptor 2} The plasmid encoding of TNF receptor protein obtained from our previous study (8). It was transferred to the $\mathrm{CHO}$ K1 cells. Glycosylated TNFR2 protein was expressed in $\mathrm{CHO}$ (Chinese hamster ovary) cells and purified by Ni-NTA His-Tag (nickel-charged nitrilotriacetic acid Histidine Tag) Affinity Chromatography and gel filtration.

\subsection{Collagen-Induced Arthritis and Medicine Treatment}

Bovine type II collagen (Chondrex, USA) was prepared according to Luo $\mathrm{Y}$ et al. at a concentration of $1 \mathrm{mg} \cdot \mathrm{mL}^{-1}$ with an equal volume of Freund's complete adjuvant (FCA) (Sigma, USA). At the beginning of the experiments (on dayl), the female Balb/c mice, were injected intradermally at the base of the tail with $1 \mathrm{~mL}$ of CII emulsion. On day 21, mice were boosted with an intraperitoneal injection of $100 \mu \mathrm{g}$ type II collagen. After the second injection of CII, all immunized mice were divided randomly into 4 groups $(n=5$ per group). The normal group was without any immunization. The control group (CIA group) was treated with PBS. The positive control group was treated with methotrexate (MTX, Sigma Aldrich, USA) (1.5 mg. $\left.\mathrm{kg}^{-1}\right)$. The treated mice were injected with TNFR2 protein intraperitoneally $\left(1 \mathrm{mg} \cdot \mathrm{kg}^{-1}\right)$. TNFR2 protein and methotrexate were administered daily during the second week from day 21 to 36 after immunization (9).

\subsection{Evaluation of Progression of Paw and Joint Edema}

From day 21 of the primary immunization, the paw widths and the circumference was measured every three days with a Vernier caliper by an independent observer without prior awareness of the experimental groups. Each paw and joint was measured three times (10).

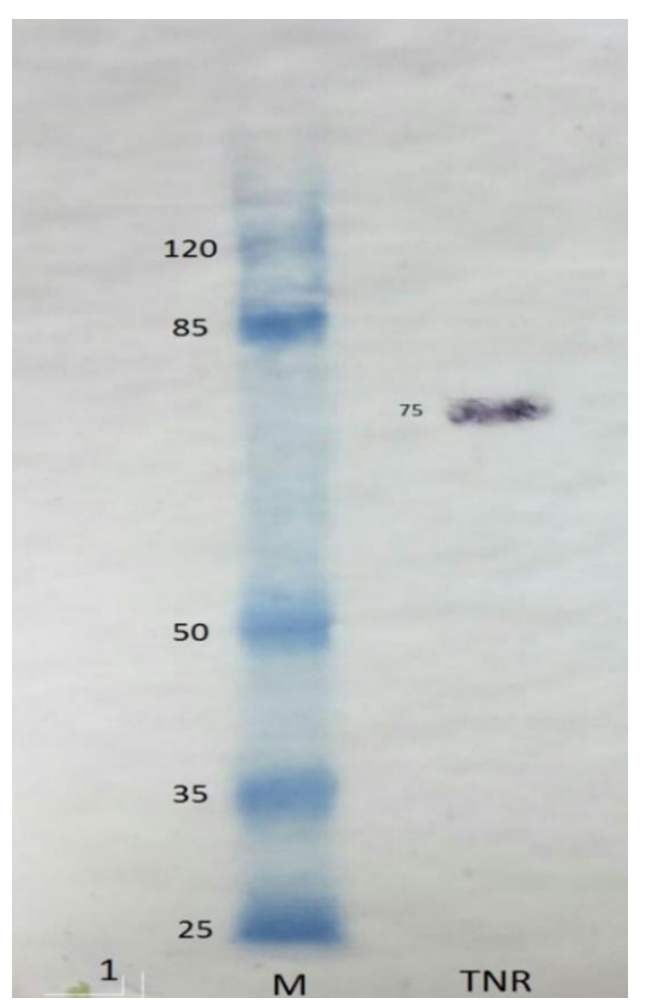

Figure 1. Western blot analysis result of the purified recombinant TNFR2 protein $(75 \mathrm{kDa})$.

\subsection{Assessment of Arthritis}

On day 37, mice were sacrificed and their right hind limbs were fixed in $4 \%$ neutral buffered formalin for 24 $\mathrm{h}$ and decalcified in $10 \%$ ethylenediaminetetraacetic acid (EDTA). Next, they were embedded in paraffin. The sections of $6 \mu \mathrm{m}$ were prepared with hematoxylin 
and eosin (H\&E) method and observed by light microscopy (11).

\subsection{Evaluation of TNF-a, IL-1 $\beta, I L-6$ and $I L-10$ Levels in Mice Serum}

Levels of total TNF- $\alpha$, IL- $1 \beta$, IL-6, and IL-10 in the serum samples of mice were analyzed by enzyme-linked immunosorbent assay (ELISA) Duoset kits (R\&D Systems Inc., USA). The concentrations of these cytokines were calculated from the standard curves.

\subsection{Statistical Analysis}

Results are reported as mean \pm S.E.M. Statistical analysis was evaluated by Student's t-test (between the control and disease), two-way (treatmentxtime) ANOVA (Analysis of Variance), and one-way ANOVA followed by Dunnett's t-test. The P-values were considered ${ }^{*}<$ $0.05,{ }^{* *} \mathrm{P}<0.01$ and ${ }^{* * *} \mathrm{P}<0.001$ for significant results.

\section{Results}

4.1. Expression and Purification of Recombinant TNFR Protein

Figure 1 demonstrates the results of the western blot analysis of the purified protein. It shows the glycosylated form of TNF receptor according to Corti A. et al report (12).

\subsection{Wide-Ranging of Paws and Knee Joints:}

The width changes of hind paws and knee joints for days 21 to 36 are presented in Tables 1,2 . The mice in treated groups with TNFR2 protein were remarkably different from those in the control group since they show a swelling reduction of paws and knee joints on day 30 .

Table 1. Effect of recombinant TNFR2 on PAW joints wellings of CIA mice. Two-way ANOVA revealed significant effects between control and treated groups

\begin{tabular}{lcccc}
\hline & Control & Disease & MTX & TNFR2 \\
\hline & Mean \pm SEM & Mean \pm SEM & Mean \pm SEM & $0.191 \pm 0.002^{* * *}$ \\
Day21 & $0.201 \pm 0.003^{* * *}$ & $0.215 \pm 0.003^{\# \# *}$ & $0.213 \pm 0.002^{* *}$ & $0.194 \pm 0.003^{* * *}$ \\
Day24 & $0.206 \pm 0.002^{* * *}$ & $0.220 \pm 0.002^{\# \# *}$ & $0.221 \pm 0.003^{* * *}$ & $0.196 \pm 0.004^{* *}$ \\
Day27 & $0.210 \pm 0.004^{* * *}$ & $0.224 \pm 0.004^{\# \#}$ & $0.214 \pm 0.004^{* *}$ & $0.13 \pm 0.001^{* *}$ \\
Day30 & $0.208 \pm 0.007^{* * *}$ & $0.225 \pm 0.007^{\# \# *}$ & $0.212 \pm 0.002^{* * *}$ & $0.191 \pm 0.001^{* * *}$ \\
Day33 & $0.206 \pm 0.005^{* * *}$ & $0.226 \pm 0.005^{\# \# *}$ & $0.209 \pm 0.002^{* * *}$ & $0.193 \pm 0.002^{* * *}$ \\
Day36 & $0.206 \pm 0.004^{* * *}$ & $0.225 \pm 0.004^{\# \# *}$ & & \\
\hline
\end{tabular}

${ }^{* *} \mathrm{P}<0.01$ compared with the Disease Group

${ }^{* * *} \mathrm{P}<0.001$ compared with the Disease Group.

\#\#\# $\mathrm{P}<0.001$ compared with the Normal Group

Table 2. Effect of recombinant TNFR2 on knee joints wellings of CIA mice. Two-way ANOVA revealed significant effects between control and treatment groups

\begin{tabular}{lcccc}
\hline & Control & Disease & MTX & TNFR2 \\
\hline & Mean \pm SEM & Mean \pm SEM & Mean \pm SEM & Mean \pm SEM \\
Day21 & $0.2744 \pm 0.003^{* *}$ & $0.289 \pm 0.003^{\# *}$ & $0.287 \pm 0.002^{* *}$ & $0.286 \pm 0.001^{*}$ \\
Day24 & $0.276 \pm 0.003^{* * *}$ & $0.291 \pm 0.0 .003^{\# \# *}$ & $0.290 \pm 0.002^{* *}$ & $0.289 \pm 0.002^{* *}$ \\
Day27 & $0.27 \pm 0.004^{* * *}$ & $0.296 \pm 0.004^{\# \# *}$ & $0.296 \pm 0.004^{* * *}$ & $0.294 \pm 0.003^{* * *}$ \\
Day30 & $0.278 \pm 0.006^{* * *}$ & $0.298 \pm 0.006^{\# \# *}$ & $0.287 \pm 0.002^{*}$ & $0.285 \pm 0.002^{* *}$ \\
Day33 & $0.279 \pm 0.006^{* * *}$ & $0.298 \pm 0.006^{\# \# *}$ & $0.286 \pm 0.004^{* *}$ & $0.285 \pm 0.003^{* *}$ \\
Day36 & $0.278 \pm 0.007^{* * *}$ & $0.299 \pm 0.007^{\# \#}$ & $0.284 \pm 0.005^{* * *}$ & $0.28 \pm 0.004^{* * *}$ \\
\hline
\end{tabular}

${ }^{*} \mathrm{P}<0.05$ compared with the disease group

${ }^{* *} \mathrm{P}<0.01$ compared with the disease group

${ }^{* * *} \mathrm{P}<0.001$ compared with the disease group

\#\# $\mathrm{P}<0.01$ compared with the normal group

\#\#\# $\mathrm{P}<0.001$ compared with the normal group

\subsection{Histological Changes}

Histological analysis displays the inhibitory traces of TNFR2 on the knee joints in the CIA mice. The nonimmunized group indicates no histopathological changes but shows normal articular cartilage (Fig. 2A). In the knee joints of the control group (CIA group) histopathological changes such as cartilage hyperplasia, synovial hyperplasia, and the formation of pannus were observed (Fig. 2B).

The group treated with recombinant protein $\left(1 \mathrm{mg} \cdot \mathrm{kg}^{-}\right.$ $\left.{ }^{1}\right)$ show reduced pathological changes in knee joints (Fig. 2C). In the group treated with MTX showed a little bit increase in synovial hyperplasia and cartilage hyperplasia in knee joints (Fig. 2D). 


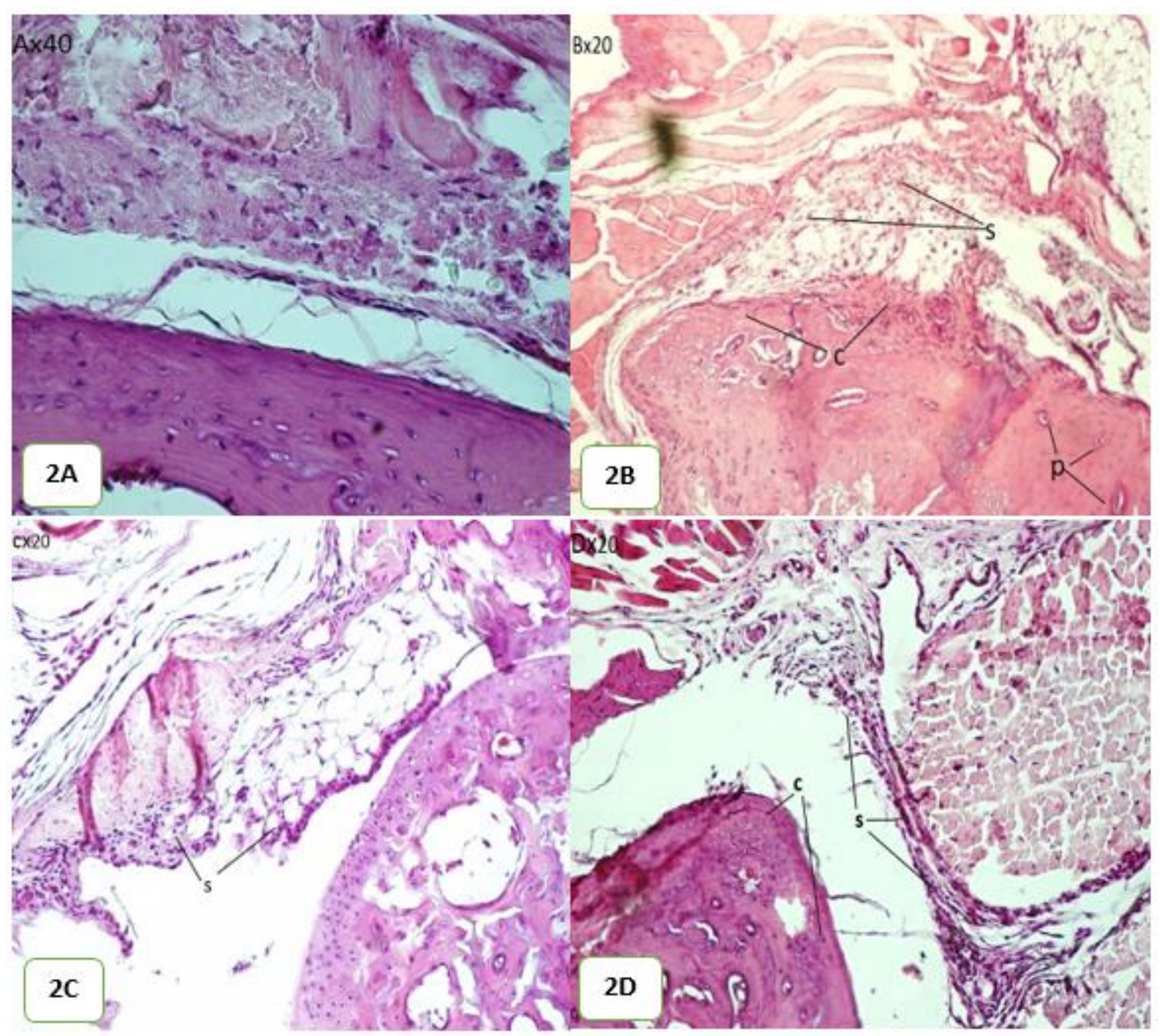

Figure 2. Histology of CIA mice: (A) The normal cartilage and synovial without inflammation (B) The joints of control mice with obvious synovial hyperplasia, cartilage hyperplasia and pannus formation (C) The joints of recombinant TNFR2 treated mice with moderated synovial hyperplasia (D) The joints of MTX treated mice with moderated synovial hyperplasia and cartilage hyperplasia c: cartilage hyperplasia; p, pannus formation and $s$, synovial hyperplasia

\subsection{Measurement of $T N F a_{\triangleleft}, I L-1 \beta, I L-6, I L-10$ in Mice Sera}

To study the levels of the inflammatory cytokines on day 36 after the first injection, the levels of TNF- $\alpha$, IL- $1 \beta$, IL6 , and IL-10 were surveyed by ELISA in blood samples. The levels of the cytokines of the normal group (Nonimmunized group), the control group (CIA group), and the groups treated with MTX and TNFR2 were evaluated.

As displayed in Figure 3, the levels of TNF- $\alpha, \mathrm{IL}-1 \beta$, and IL-6, in the serum of the CIA group were remarkably higher than those in the normal group $(\mathrm{P}<0.01)$.

It was shown that the levels of TNF- $\alpha$, IL- $1 \beta$, and IL- 6 in groups treated with recombinant protein and MTX were clearly lower than those of CIA group $(\mathrm{P}<0.01, \mathrm{P}$ $<0.001)$.

As shown in Figure 3, the level of TNF-10 in the serum of the CIA group was lower than that in the normal group $(\mathrm{P}<0.01)$. In comparison, the level TNF-10 in the serum of the groups treated with the recombinant protein and MTX was clearly higher than the CIA group $(\mathrm{P}<0.01)$.

\section{Discussion}

RA is an advanced chronic disease in human due to antigen precipitation in the joints. This disease is composed of the pannus formation, synovial cellular hyperplasia, infiltrations of the inflammatory cells, autoantibody production (rheumatoid factor and anticitrullinated protein antibody (ACPA), cartilage and bone destruction, and many different cytokines (13, 14).TNF- $\alpha$ a cytokines in the synovial fluid of RA, is mostly produced by activated immune cells in reaction to infections and tissue damage. TNF- $\alpha$ is upstream to the proinflammatory cytokines network $(6,15)$. One of the main properties of $\mathrm{TNF} \alpha$ is inducing secretion of pro-inflammatory cytokines such as IL-1 and IL-6. 

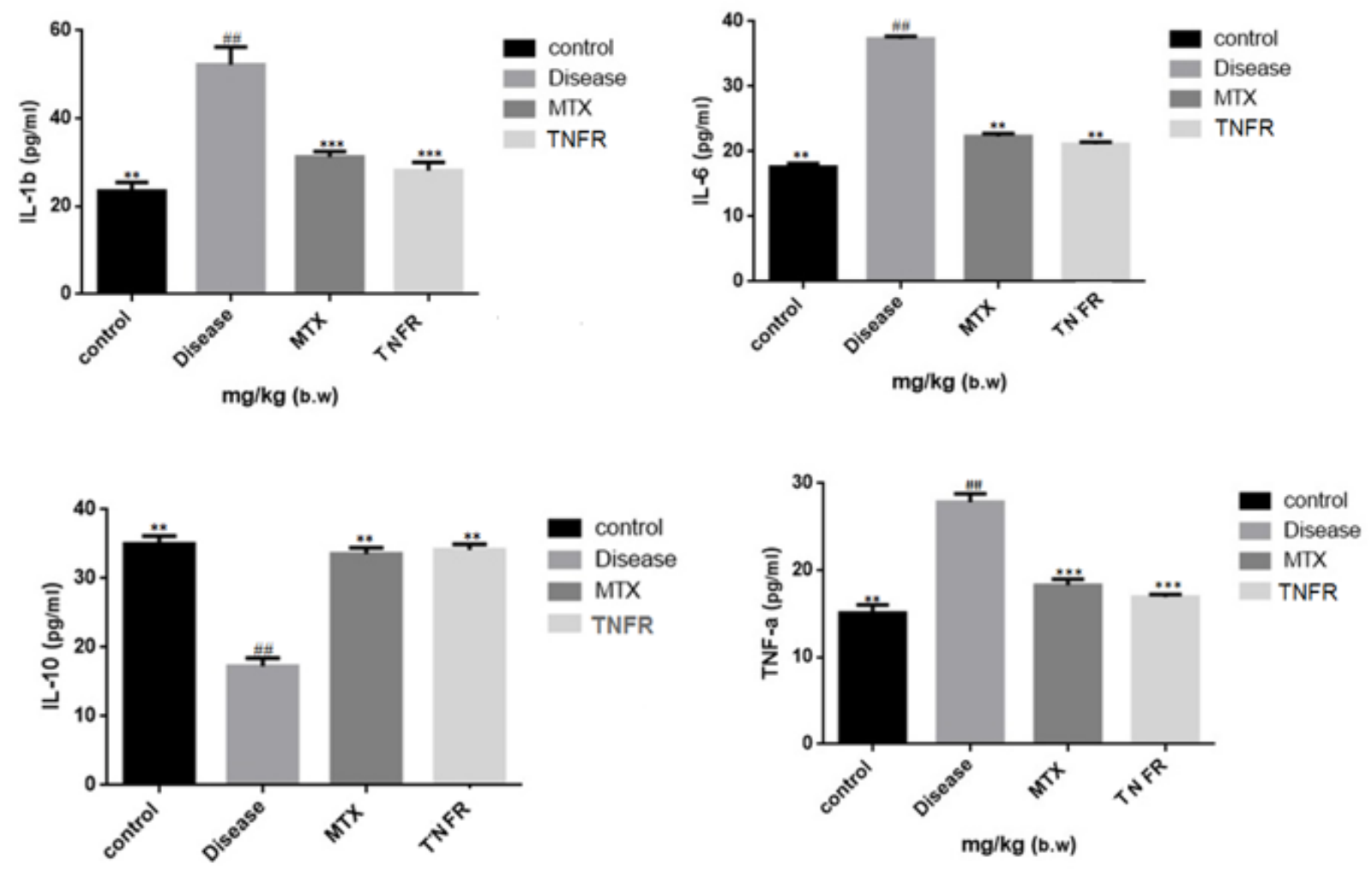

Figure3. Level of serum TNF $\alpha$, IL1b. IL6 and IL10 in CLA mice (mean \pm S.E.M, $n=5$ ). Effect of recombinant TNFR2 on the regulation of the serum inflammatory cytokines in CLA mica

** $\mathrm{P}<0.01$ compared with the control group

*** $\mathrm{P}<0.001$ compared with the control group

\#\# $\mathrm{P}<0.01$ compared with the normal group

It has been also proven that cytokines like TNF- $\alpha$, IL- $1 \beta$, and IL- 6 are produced in high quantities in all of the rheumatoid synovial membrane samples (16). The purpose of the present study was to investigate the therapeutic value of recombinant TNFR2 on CIA in $\mathrm{balb} / \mathrm{c}$ mice. Our results showed that paw and knee edema in CIA decreased with the administration of the recombinant TNFR2 protein to CIA mice during a twoweek period. On the other hand, histological analysis revealed that this protein decreases the histopathological signs such as synovial hyperplasia, pannus formation, and cartilage hyperplasia, and bone erosion. The results also showed that recombinant protein affects the cytokines production by immune cells. This effect might be due to the fact that TNF $\alpha$ and IL-1 are critical cytokines in the procedure of chronic joint inflammation and the eventual destruction at the junction of the synovial lining in the joint capsule with the cartilage and bone. IL-1, IL6, and TNF $\alpha$ pleiotropic produced predominantly by macrophages. IL-1 and $\mathrm{TNF} \alpha$ definitely induced destructive matrix metalloproteinases, such as MMP-1 (collagenase) and MMP-3 (stromelysin) that, in turn, cause cartilage damage and induce bone resorption in in vitro cultures. Moreover, IL-6 may prevent bone synthesize and affect bone resorption through its stimulatory in osteoclasts
(17). IL-6 is recognized as a pro-inflammatory cytokine that is activated after binding to a receptor. This protein affects the maturation and activation of $\mathrm{B}$ and $\mathrm{T}$ cells, macrophages, osteoclasts, and chondrocytes and also plays a crucial role in bone and cartilage degradation. IL10 is an anti-inflammatory and immunosuppressive cytokine that has been shown to regulate endogenous proinflammatory cytokine synthesis in RA synovial tissue. In this work, neutralization of endogenously produced IL-10 in the RA synovial membrane cultures resulted in a remarkable increase in the levels of proinflammatory cytokines TNF- $\alpha$ and IL- $1 \beta$. IL-10 has also been shown to suppress the synthesis of IL- $1 \beta$, IL6 , and TNF- $\alpha(18,19)$.

$\mathrm{TNF} \alpha$ as a therapeutic target plays a key role in rheumatoid arthritis treatment. This theory has been successfully examined in animal models with, collageninduced arthritis and the assays have paved the way for clinical trials of anti-TNF therapy in patients with rheumatoid arthritis. Since TNFR2 plays a crucial role in tissue regeneration and immune regulation. The ligands that successfully activate TNFR2 have been used as new therapeutics for treatment inflammatory autoimmune diseases such as RA (7).

Studies have demonstrated that two different types of soluble TNF receptor (sTNFR) and trivalent soluble 
TNF Receptor2 or some anti-tumor necrosis factor antibodies (fusing a sTNFR with the Fc region of IgG1) prevent collagen arthritis in mice. We used insoluble recombinant TNFR2 (total TNFR2) for the treatment of mice CIA.

As TNFa is homotrimer and it appears that TNFR2 monomers to spontaneously form a hemotrique ligand (biologically active protein) that may have suitable docking sites and theoretically has a higher affinity to TNFa. This can offer a molecular basis for the block anti-TNF drug in treating RA. Additionally, it has been recently shown that the expression of TNFR2 on hematopoietic cells decreases the intensity of TNFdriven arthritis. Finally, regulatory $\mathrm{T}$ cell (Treg) has appeared as likely mediator of the anti-inflammatory factors of TNFR2 $(20,21)$.

According to Köhne's study the glycosylation of secreted recombinant TNFR-IgG1 fusion protein impacted its intracellular transport and secretion of other variants with binding properties similar to the fully glycosylated protein (22).

We used recombinant TNFR2 intra peritoneal administrated for fourteen days without interruption from the day 21 to 36 , Based on our results, our approach, could effectively improve clinical symptoms of mice i.e., showed by the dramatic decrease in inflammatory swelling in the knee joints and the paws, and the reduced pathological changes in knee joints and this recombinant protein remarkably decreased the infiltration of inflammatory cells into the synovial area and prevented bone and cartilage from erosion. The serum levels of cytokines TNF- $\alpha$, IL- $1 \beta$, and IL-6 significantly reduced in groups treated with the recombinant TNFR2. Our findings suggest that complete TNFR2 in rheumatic arthritis in mice and suggests a suitable strategy is for anti-inflammatory therapy in RA. However, we recommend further studies using administrate different doses of this recombinant protein to CIA.

\section{Conclusions}

The spite of the different types of drugs usage for reducing the inflammation in patients with Rheumatoid and being many reports about their side effects, our findings show that administration of recombinant TNF receptor has blocked or protected joints from progressive damages. This study suggests that the antiarthritic effectiveness of TNFR2 will repress the symptoms of rheumatoid arthritis. Besides, it seems that TNFR2 is a strong candidate for the treatment of the RA disease.

\section{Acknowledgments}

This article was extracted from Shahla Korani's Ph.D. thesis conducted in the Cellular \& Molecular Biology Research Center of Shahid Beheshti University of Medical Sciences. The study was funded by the deputy of Shahid Beheshti University of Medical Sciences (grant number 5061) adhering to the ethics code IR.SBMU.RAM. REC.1394.82.7.

\section{References}

1. Scott DL. Biologics-based therapy for the treatment of rheumatoid arthritis. Clin Pharmacol Ther. 2012;91(1):30-43. doi: 10.1038/clpt.2011.278 pmid: 22166850

2. Chunxia C, Peng Z, Huifang P, Hanli R, Zehua H, Jizhou W. Extracts of Arisaema rhizomatum CEC Fischer attenuate inflammatory response on collageninduced arthritis in BALB/c mice. J Ethnopharmacol 2011;133(2):573-582.

3. Lim H, Lee SH, Lee HT, Lee JU, Son JY, Shin W, et al. Structural Biology of the TNFalpha Antagonists Used in the Treatment of Rheumatoid Arthritis. Int J Mol Sci. 2018;19(3):768. doi: 10.3390/ijms19030768 pmid: 29518978

4. Faustman D, Davis M. TNF receptor 2 pathway: drug target for autoimmune diseases. Nat Rev Drug Discov. 2010;9(6):482-493. doi: 10.1038/nrd3030 pmid: 20489699

5. Fischer R, Kontermann RE, Maier O. Targeting sTNF/TNFR1 signaling as a new therapeutic strategy. Antibodies. 2015;4(1):48-70.

6. Puppo F, Murdaca G, Ghio M, Indiveri F. Emerging biologic drugs for the treatment of rheumatoid arthritis. Autoimmun Rev. 2005;4(8):537-541. doi: 10.1016/j.autrev.2005.04.016 pmid: 16214092

7. Fischer R, Marsal J, Gutta C, Eisler SA, Peters N, Bethea $\mathrm{JR}$, et al. Novel strategies to mimic transmembrane tumor necrosis factor-dependent activation of tumor necrosis factor receptor 2. Sci Rep. 2017;7(1):6607. doi: 10.1038/s41598-017-06993-4 pmid: 28747780

8. Korani S, Kaszadeh M, Yarian F, Bandehpour M. The Expression of Human Tumor Necrosis Factor Receptor (Htnfr) in the CHO Cell Line. Int J Molecul Biol 2017;2(3):00021.

9. Luo Y, Liu M, Xia Y, Dai Y, Chou G, Wang Z. Therapeutic effect of norisoboldine, an alkaloid isolated from Radix Linderae, on collagen-induced arthritis in mice. Phytomedicine. 2010;17(10):726-731. doi: 10.1016/j.phymed.2010.01.013 pmid: 20363113

10. Lam FF, Wong HH, Ng ES. Time course and substance $P$ effects on the vascular and morphological changes in adjuvant-induced monoarthritic rats. Int Immunopharmacol. 2004;4(2):299-310. doi: 10.1016/j.intimp.2004.01.009 pmid: 14996421

11. Luo X, Li LL, Zhang SS, Lu JL, Zeng Y, Zhang HY, et al. Therapeutic effects of total coumarins from Urtica dentata Hand on collagen-induced arthritis in Balb/c mice. J Ethnopharmacol. 2011;138(2):523-529. doi: 10.1016/j.jep.2011.09.050 pmid: 22001857

12. Corti A, D'Ambrosio F, Marino M, Merli S, Cassani G. Identification of differentially glycosylated forms of the soluble p75 tumor necrosis factor (TNF) receptor in human urine. Eur Cytokine Netw. 1995;6(1):29-35. pmid: 7795172

13. McInnes IB, Schett $\mathrm{G}$. The pathogenesis of rheumatoid arthritis. N Engl J Med. 2011;365(23):2205-2219. doi: 10.1056/NEJMra1004965 pmid: 22150039 
14. Rein P, Mueller RB. Treatment with Biologicals in Rheumatoid Arthritis: An Overview. Rheumatol Ther. 2017;4(2):247-261. doi: 10.1007/s40744-017-0073-3 pmid: 28831712

15. Takeuchi T, Amano K, Kameda H, Abe T. Anti-TNF biological agents in rheumatoid arthritis and other inflammatory diseases. Allergol Int 2005;54(2):191202.

16. Yang K, Tong L, Chen C, Zhang P, Pi H, Ruan H, et al. Therapeutic effects of extracts from Radix Toddaliae Asiaticae on collagen-induced arthritis in Balb/c mice.J Ethnopharmacol. 2013;146(1):355-362. doi: 10.1016/j.jep.2013.01.004 pmid: 23337745

17. Feldmann M, Brennan FM, Maini RN. Role of cytokines in rheumatoid arthritis. Annu Rev Immunol. 1996;14(1):397-440. doi: 10.1146/annurev.immun ol.14.1.397 pmid: 8717520

18. Kunz M, Ibrahim SM. Cytokines and cytokine profiles in human autoimmune diseases and animal models of autoimmunity. Mediators Inflamm. 2009;2009:979258. doi: $10.1155 / 2009 / 979258$ pmid: 19884985
19. Quattrocchi E, Dallman MJ, Dhillon AP, Quaglia A, Bagnato G, Feldmann M. Murine IL-10 gene transfer inhibits established collagen-induced arthritis and reduces adenovirus-mediated inflammatory responses in mouse liver. J Immunol. 2001;166(10):5970-5978. pmid: 11342612

20. Cui X, Chang L, Li Y, Lv Q, Wang F, Lin Y, et al. Trivalent soluble TNF Receptor, a potent TNF-alpha antagonist for the treatment collagen-induced arthritis. Sci Rep. 2018;8(1):7327. doi: 10.1038/s41598-01825652-w pmid: 29743640

21. Bluml S, Scheinecker C, Smolen JS, Redlich K. Targeting TNF receptors in rheumatoid arthritis. Int Immunol. 2012;24(5):275-281. doi: 10.1093/intimm /dxs047 pmid: 22457216

22. Kohne C, Johnson A, Tom S, Peers DH, Gehant RL, Hotaling TA, et al. Secretion of glycosylation site mutants can be rescued by the signal/pro sequence of tissue plasminogen activator. $J$ Cell Biochem. 1999;75(3):446-461. pmid: 10536368 